\title{
COMMENTARY
}

\section{Deeper understanding of mechanisms contributing to sepsis-induced myocardial dysfunction}

Keith R Walley

See related research by Koesters et al., http://ccforum.com/content/18/2/R54

\begin{abstract}
The inflammatory response of sepsis results in organ dysfunction, including myocardial dysfunction. Myocardial dysfunction is particularly important in patients with severe septic shock who progress to a hypodynamic pre-terminal phase. Multiple aspects of this septic inflammatory response contribute to the pathogenesis of decreased ventricular contractility. Inflammatory cytokines released by inflammatory cells contribute as does nitric oxide released by vascular endothelium and by cardiomyocytes. Endotoxins and other pathogen molecules induce an intramyocardial inflammatory response by binding Toll-like receptors on cardiomyocytes that then signal via NF-kB. These processes alter cardiomyocyte depolarization and, therefore, contractility. The particular role of the cardiomyocyte sodium current has not been characterized. Now new information suggests that the septic inflammatory response impairs normal depolarization by altering the cardiomyocyte sodium current. This results in decreased ventricular contractility. This is important because new targets for therapeutic intervention can be considered and new approaches to evaluation of this problem can be contemplated.
\end{abstract}

In the previous issue of Critical Care, Koesters and colleagues [1] contribute to our understanding of sepsisinduced ventricular dysfunction. Sepsis-induced ventricular dysfunction is increasingly recognized as a contributor to cardiovascular dysfunction during septic shock [2]. Vasodilation induced by nitric oxide (NO) and other mediators figures prominently in the hypotensive distributive shock circulation induced by sepsis. But additional sepsis-induced ventricular dysfunction can result in an inadequate cardiac output response to decreased afterload, resulting in preterminal hypodynamic septic shock [3]. It could be argued that decreased ventricular contractility during sepsis is not a major issue in hyperdynamic septic shock characterized by an elevated cardiac output. Yet death due to septic shock is characterized by onset and progression of a hypodynamic phase that, in the volume-resuscitated patient, can only be explained by myocardial dysfunction. So ventricular dysfunction may be less important in septic shock survivors but myocardial dysfunction of sepsis may well be the deciding factor in early mortality of septic shock.

Correspondence: Keith.Walley@hli.ubc.ca

Centre for Heart Lung Innovation, St Paul's Hospital, University of British Columbia, Vancouver, BC V6Z 1Y6, Canada
The septic inflammatory response involves a surprising number of intersecting pathways, many of which contribute to ventricular dysfunction. Pathogen-associated molecular patterns (PAMPs) released by infecting organisms bind innate immune receptors on inflammatory cells (for example, monocytes, neutrophils) but also bind innate immune receptors in the heart [4] and within the coronary macroand micro-circulation. For example, cardiomyocytes express a variety of Toll-like receptors that, when bound by a PAMP, induce intracellular signaling via NF- $\mathrm{KB}$, which then induces an intra-myocardial inflammatory response [5]. In addition, early pro-inflammatory cytokines, such as tumor necrosis factor- $\alpha$ and interleukin- $1 \beta$ [6], released by similarly activated inflammatory cells contribute in part by increased production of NO [7]. NO can decrease ventricular contractility by activating guanylate cyclase, which results in decreased intracellular calcium concentration. A variety of inflammatory mediators induce expression of cellular adhesion molecules by endothelial cells of the coronary microcirculation and even by cardiomyocytes. Ligation of cardiomyocyte-expressed intracellular adhesion molecule (ICAM)- 1 by ligands such as fibrinogen result in decreased contractility [8]. Chemotactic cytokines such as macrophage inflammatory protein 2 are also expressed by 
activated cardiomyocytes. Increased production of chemokines leads to retention of neutrophils and other leukocytes in the coronary circulation and extravasation of these inflammatory cells into the myocardium. Contact between leukocytes and cardiomyocytes mediated by ICAM-1 and other adhesion molecules also results in a decrease in cardiomyocyte contractility [9]. Ligation of cardiomyocyteexpressed ICAM-1 signals via the cortical actin cytoskeleton resulting in altered intracellular calcium flux [10].

Alteration of intracellular calcium flux is a fundamental later step in the mechanistic pathway to decreased cardiomyocyte and ventricular contractility. In the previous issue of Critical Care Koesters, Engisch and Rich investigate the role of cardiomyocyte sodium current [1]. Alterations in sodium current have been observed in skeletal muscle and in nerves so these authors tested the hypothesis that similar abnormalities may be observed in myocardial tissue. Papillary muscles excised from septic rats 1 day after cecal ligation and puncture were studied. They found that action potentials of papillary muscles from septic rats differed substantially from those of controls. Action potential magnitude was decreased, the threshold was increased, and the rate of rise was decreased. Notably, the threshold and rate of rise of the action potential are known to be heavily influenced by the sodium channel. To put these results into context, these investigators compared these intraabdominal sepsis observations to those where the sodium channel was inhibited using tetrodotoxin. There were similarities between sepsis-induced changes and tetrodotoxininduced changes. Both intra-abdominal sepsis and a comparable concentration of tetrodotoxin (similar effects on the action potential) decreased papillary muscle force by more than half. The authors conclude that the sepsisinduced changes in sodium channel function could contribute substantially to decreased ventricular contractility during sepsis.

To determine whether other channel currents could have contributed, additional characteristics of the septic action potential were measured. There was no significant change in resting potential, a property influenced by non-voltage gated $\mathrm{K}$ channels. Membrane resistance and action potential duration decreased only slightly in septic action potentials, suggesting a limited role, at best, for voltage gated $\mathrm{K}$ and $\mathrm{Ca}$ channels. Inhibition of the L-type $\mathrm{Ca}$ current reduced contractility, as expected.

Thus, these novel results raise the possibility that sepsis-induced decreases in sodium channel flux may contribute to ventricular dysfunction during sepsis. This conjecture is based on similarity between sepsis-induced and tetrodotoxin-induced changes and therefore is not mechanistic proof of a role of the sodium channel. Nevertheless, moving the focus of investigation towards intracellular signaling expands our knowledge of potential mechanistic pathways contributing to myocardial dysfunction of sepsis. This is important because new targets for therapeutic intervention can be considered and new approaches to evaluation of this problem can be contemplated, possibly even based on surface measurements of electrical properties of the heart.

\section{Abbreviations}

ICAM: Intracellular adhesion molecule; NF-kB: Nuclear factor kappa B; NO: Nitric oxide; PAMP: Pathogen-associated molecular pattern.

\section{Competing interests}

The author declares that he has no competing interests.

\section{Acknowledgements}

Support: Canadian Institutes of Health Research.

\section{Published: 01 May 2014}

\section{References}

1. Koesters A, Engisch KL, Rich MM: Decreased cardiac excitability secondary to reduction of sodium current may be a significant contributor to reduced contractility in a rat model of sepsis. Crit Care 2014, 18:R54.

2. Charpentier J, Luyt CE, Fulla Y, Vinsonneau C, Cariou A, Grabar S, Dhainaut JF, Mira JP, Chiche JD: Brain natriuretic peptide: a marker of myocardial dysfunction and prognosis during severe sepsis. Crit Care Med 2004, 32:660-665.

3. Maeder M, Fehr T, Rickli H, Ammann P: Sepsis-associated myocardial dysfunction: diagnostic and prognostic impact of cardiac troponins and natriuretic peptides. Chest 2006, 129:1349-1366.

4. Boyd JH, Mathur S, Wang Y, Bateman RM, Walley KR: Toll-like receptor stimulation in cardiomyoctes decreases contractility and initiates an NFkappaB dependent inflammatory response. Cardiovasc Res 2006, 72:384-393.

5. Li X, Luo R, Chen R, Song L, Zhang S, Hua W, Chen H: Cleavage of IkappaBalpha by calpain induces myocardial NF-kappaB activation, TNFalpha expression, and cardiac dysfunction in septic mice. Am J Physiol Heart Circ Physiol 2014, 306:H833-H843.

6. Kumar A, Thota V, Dee L, Olson J, Uretz E, Parrillo JE: Tumor necrosis factor alpha and interleukin 1 beta are responsible for in vitro myocardial cell depression induced by human septic shock serum. J Exp Med 1996, 183:949-958.

7. Finkel MS, Oddis CV, Jacob TD, Watkins SC, Hattler BG, Simmons RL: Negative inotropic effects of cytokines on the heart mediated by nitric oxide. Science 1992, 257:387-389.

8. Boyd JH, Chau EH, Tokunanga C, Bateman RM, Haljan G, Davani EY, Wang Y, Walley KR: Fibrinogen decreases cardiomyocyte contractility through an ICAM-1-dependent mechanism. Crit Care 2008, 12:R2.

9. Davani EY, Boyd JH, Dorscheid DR, Wang Y, Meredith A, Chau E, Singhera GK, Walley KR: Cardiac ICAM-1 mediates leukocyte-dependent decreased ventricular contractility in endotoxemic mice. Cardiovasc Res 2006, 72:134-142.

10. Davani EY, Dorscheid DR, Lee CH, van Breemen C, Walley KR: Novel regulatory mechanism of cardiomyocyte contractility involving ICAM-1 and the cytoskeleton. Am J Physiol Heart Circ Physiol 2004, 287:H1013-H1022.

\section{$10.1186 / \mathrm{cc} 13853$}

Cite this article as: Walley: Deeper understanding of mechanisms contributing to sepsis-induced myocardial dysfunction. Critical Care 2014, 18:137 